\title{
Aggregation operators and models
}

\author{
Vicenç Torra* \\ Institut d'Investigació en Intelligència Artificial-CSIC, Campus UAB, E-08193 Bellaterra, Catalonia, Spain
}

Available online 20 June 2005

\begin{abstract}
This paper gives an overview of the field of aggregation operators. Current research lines are described focusing on those related with the process of building real applications.
\end{abstract}

(C) 2005 Elsevier B.V. All rights reserved.

Keywords: Aggregation operators; Data modeling; Applications

\section{Introduction}

Aggregation operators, in particular, and data fusion methods, in general, have received a lot of attention in the last years. New methods have been developed and sound theoretical results have been obtained. Research results establish relationships between methods, describe their basic properties and define a roadmap for their use in practical applications. Such results are rooted in an old research trend on mechanisms to aggregate information. Ramon Llull, Nicolas di Cusa, Condorcet and Borda are some, to name a few, forerunners in this area [3]. Others such as Vitali, who defined the functional currently known as Choquet integral, in 1925, are not so known but also contributed to the development of the field. One may consider that current research follows the imprints of e.g. Cauchy, De Finetti, Aczél; and in the particular framework of fuzzy integrals Sugeno and Choquet.

The current theoretical interest to define a good roadmap for aggregation operators is tightly related with an increasing practical interest on using them for building applications. The amount of stored information is increasing day by day, and, thus, summarization mechanisms for reducing the burden of information as well as decision mechanisms to make timely decisions on the basis of such overwhelming data are

\footnotetext{
* Tel.: +34935809570; fax: +34935809661.

E-mail address: vtorra@iiia.csic.es.
} 
needed. Aggregation operators are one of the basic bricks for building such systems. This causes that aggregation operators are currently in use in a large number of applications being very much different in nature. Aggregation operators are used in systems ranging from bioinformatics to information retrieval, from robotics to computer vision applications. Also, the information types that can be aggregated are large.

From a technical point of view, theoretical issues have reached in recent years a good level of maturity. For example, characterizations have been obtained for a large number of aggregation operators. See e.g. [5,2] for a good account on such results. Nevertheless, aggregation methods embedded in applications are still rather simple and do not exploit much of the most powerful operators. Instead, they usually use simple mechanisms (arithmetic or weighted mean, median and the like) and when more complex operators are used (as fuzzy integrals), their parameters are particularized so that the expressive power of the operator is radically diminished. In fact, this is the case of most practical applications of Choquet integrals that use particular classes of fuzzy measures that are rather simple from their expressive power point of view. For example, some of such applications use Sugeno $\lambda$-measures that are uniquely described using $N$ values while arbitrary fuzzy measures require $2^{N}$.

In this paper we outline some of the open research issues with respect to aggregation operators focusing on the requirements for their embedding in real applications. Although some theoretical issues are still open and they can be further developed, we focus here on those aspects that we think that are more directly related with the construction of applications.

\section{Model building}

When a new problem is encountered in which decision or summarization plays a role, the construction of a suitable model is not an easy task. This is so because several alternatives should be taken into account (e.g. operators, parameters). The modeling problems can be roughly classified at three different levels of abstraction. We review below such problems following a top-down description (from the most general problem to more specific ones).

Architecture construction: How aggregation operators and fusion mechanisms are integrated among themselves and with the system so that correct decisions and appropriate summaries are computed and used by the system to achieve its goals. For example, what is the appropriate information flow to integrate user preferences, knowledge-based systems' outcomes and search engine results to obtain a list of relevant web pages.

Function/operator selection: Once a generic architecture is built, the concrete fusion mechanisms have to be selected, e.g., which is the best aggregation operator to fuse lists of web pages.

Parameter learning: Aggregation operators are typically parametric (e.g., weights in the weighted mean and fuzzy measures in fuzzy integrals). Prior to the application of the model, parameters have to be fixed so that the system behaves in an appropriate way, e.g., which fuzzy measure should be used for combining ratings when we use a Choquet integral.

These three modeling problems introduce three different kinds of research topics. We review them below.

Parameter learning: In the last years, several methods for parameter determination have been developed. Approaches can be distinguished according to the available information. One case is when there is an expert participating in the modeling process. This would be the case of Saaty's Analytical Hierarchy 
Process (AHP) [8]. In AHP, it is assumed that we can interview a user and from his answers we extract the weights of a weighted mean. A similar approach was introduced by O'Hagan [7]. In his work, parameters are extracted from the so-called orness or compensation. In the case that several alternative parameters are consistent with the orness maximum dispersion is also required. In both approaches, it is assumed the existence of someone that supplies some critical information to the system, and from such information parameters are extracted. Another research trend is to determine the parameters from examples. In this case, the typical situation is that we have a set of (input, output) pairs and an operator $\mathbb{C}$ with some parameter $P$ to fit, then the parameter is selected so that the divergence between the output of $\mathbb{C}_{P}($ input $)$ and output is minimized. From the machine learning perspective, all these approaches correspond to supervised learning methods, i.e., the model is learnt from examples.

Recently, an alternative approach was proposed by Kojadinovic [4]. This approach, following machine learning jargon, corresponds to unsupervised learning. This is a promising alternative as it overcomes the limitation of supervised approaches and does not need that examples include an outcome of their evaluation. Following with the analogy with machine learning, another line of research would be reinforcement learning. In this case, only a reward/penalty would be used by the learning algorithm.

The consideration of machine learning research and its application in the setting of aggregation operators poses interesting questions and opens new lines of research. One example is the consideration of the bias-variance problem.

Function/operator selection: Selection of the operator is another important issue. At present much research has been devoted to the characterization of existing methods. As a result, research methods have been classified, families of methods have been distinguished, and generalization/particularization relationships have been established between operators. For example, it is known that the Choquet integral generalizes the weighted mean and that the Sugeno integral generalizes weighted maximum and weighted minimum. Characterization results permit to know which is the set of functions that satisfy a given set of properties.

The development of algorithms and methodologies to determine (automatically) the suitable function for a given problem is a new topic of research (see e.g. [1]). Both supervised and unsupervised approaches could also be applied here.

Architecture determination: The topic of architecture determination encompasses two different aspects. On the one hand, we have the more general aspect of architectures for information integration. That is, an architecture that distinguishes the steps of: data acquisition, preprocessing, fusion and the final execution of an action. On the other hand, we can consider architectures of operators, i.e., complex/composite models defined in terms of aggregation operators. Hierarchical models (as the two-step Choquet integrals) are examples of such architectures. Here we will consider this second topic.

Recent results have shown that hierarchical models based on aggregation operators can approximate any arbitrary function at the desired level of detail $[9,6]$. Nevertheless, the flexibility of such models is at the expenses of a higher cost in the process of defining the architecture and its parameters. Note that for defining such models we should have detailed information about the sources, and about which data can be combined together to get meaningful partial results. Due to this, the definition of the architecture is a difficult problem, and such architecture later requires the particularization of the aggregation operators and of their parameters.

Nowadays research in this area is focused on the properties of the models (as in the above-mentioned work [6]). Some applications of composite models have also developed in the literature. Nevertheless methodologies for building such composite models and for determining both the operators and the 
parameters are required. This research links with other research on composite models as hierarchical fuzzy systems [10].

\section{Conclusions}

In this paper we have reviewed some of the research topics of aggregation operators related with the process of building applications. Other research topics not detailed here are also of interest in this setting. One of them is dynamic aggregation operators.

Current research on aggregation operators is mainly focused on time independent operators. Accordingly, as time is not taken into account, operators and weights are usually kept constant on the whole operational time of the system. The more systems use operators to fuse information and the longer their life time, the more need for dynamic functions we have. Dynamic weights is an alternative that can be used to model dynamic behaviors.

\section{References}

[1] G. Beliakov, J. Warren, Appropriate choice of aggregation operators in fuzzy decision support systems, IEEE Trans. Fuzzy Systems 9 (6) (2001) 773-784.

[2] T. Calvo, A. Kolesárová, M. Komorníková, R. Mesiar, Aggregation operators: properties, classes and construction methods, in: T. Calvo, G. Mayor, R. Mesiar (Eds.), Aggregation Operators, Physica-Verlag, 2002, pp. 3-104.

[3] G. Hägele, F. Pukelsheim, Llull's writings on electoral systems, Studia Lulliana 41 (2001) 3-38.

[4] I. Kojadinovic, Unsupervised aggregation by the Choquet integral based on entropy functionals: application to the evaluation of students, Lecture Notes in Artificial Intelligence, vol. 3131, 2004, pp. 163-174.

[5] J.-L. Marichal, Aggregation operators for multicriteria decision aid, Ph.D. Dissertation, Institute of Mathematics, University of Liège, Liège, Belgium, 1998.

[6] T. Murofushi, Y. Narukawa, A characterization of multi-level discrete Choquet integral over a finite set (in Japanese), Proc. of Seventh Workshop on Evaluation of Heart and Mind, 2002, pp. 33-36.

[7] M. O'Hagan, Aggregating template or rule antecedents in real-time expert systems with fuzzy set logic, in: Proc. of the 22nd Annu. IEEE Asilomar Conf. on Signals, Systems and Computers, Pacific Grove, CA, 1988, pp. 681-689.

[8] T.L. Saaty, The Analytic Hierarchy Process, McGraw-Hill, New York, 1980.

[9] V. Torra, On some relationships between hierarchies of quasi-arithmetic means and neural networks, Internat. J. Intel. Systems 14 (11) (1999) 1089-1098.

[10] E. Tunstel, M.A.A. Oliveira, S. Berman, Fuzzy behavior hierarchies for multi-robot control, Internat. J. Intel. Systems 17 (2002) 449-470. 